\title{
Graphene/Polypyrimidine Composite Film Modified Electrode for Electrochemical Determination of Sunset Yellow
}

\author{
FU Jua ${ }^{a}$ TAN Xiaohong ${ }^{b}$ and SONG Xinjian ${ }^{c}$
}

School of Chemical and Environmental Engineering, Hubei University for Nationalities, Enshi, China

a1186914340@qq.com, bhbxhtan@163.com, cwhxjsong@163.com

Keywords: Graphene; 4-amino-5-cyano-2-mercapto-6-phenylpyrimidine; electrochemical sensor; sunset yellow.

Abstract. Graphene nanosheets (GN) were efficiently prepared through one-step liquid-phase exfoliation of graphite powder, and then used to modify the surface of glassy carbon electrode (GCE) by drop to form a GN/GCE nanointerface. Subsequently, 4-amino-5-cyano-2-mercapto-6-phenylpyrimidine (ACMP) was electropolymerized onto the GN/GCE surface to provide a composite film-modified electrode (pACMP/GN/GCE). Finally, a sensitive and rapid electrochemical sensor was successfully constructed for the determination of sunset yellow.

\section{Introduction}

Graphene, a single atomic layer of $\mathrm{sp}^{2}$-bonded carbon atoms arranged in a honeycomb lattice, exhibits large specific surface area, high thermal conductivity, and extraordinary electronic transport properties [1]. Graphene have been applied in various fields because of these exciting properties [2]. Recently, graphene-based composites have been highly concerned in widespread applications, ranging from environmental science, energy conversion, to sensing [3]. Conducting polymers with high conductivity, thermal and environmental stability [4], have attracted much attention in the field of electrochemistry, and have been widely used to construct modified electrodes [5]. Particularly, it has been demonstrated that conducting polymer/GN nanomaterials, used as sensing platforms, display remarkable electro- chemical performance. As is well-known, chemical oxidation is usually applied to synthesize polymeric films, however, electrochemical polymerization is a convenient and feasible way for in-situ preparation of polymeric films [6]. Moreover, the morphology and thickness of the resulting layers can be easily altered by controlling the experimental parameters, such as concentration, potential and deposition time. Then it could be expected that the integration of polypyrimidine and GN would not only significantly improve the electrocatalytic properties of substrates but also increase the reaction rate.

Monitoring of synthetic dyes in foods is very important owing to their potential harmful effects on human beings. Sunset yellow (SY) is a synthetic organic food dye found in common food products, such as softdrinks, baked products, candies, beverages, and dairy products. Therefore, its sensitive, rapid and convenient detection is highly desired. Different graphene-based modified electrodes, such as graphene/ mesoporous $\mathrm{TiO}_{2}$ composite-coated electrode [7], graphene layer-wrapped phosphotungstic acid (PTA) hybrid modified GCE [8], and $\beta$-cyclodextrin/poly(diallyldimethylammonium chloride)/graphene com- posite modified GCE [9], have been developed for the electrochemical determination of SY.

In this work, exfoliated graphene was easily obtained through short-time mild ultrasonication of graphite powder in low-toxic $N, N$-dimethylformamide (DMF) with the assistance of sodium citrate. The resulting GN suspension was then used to modify the surface of glassy carbon electrode (GCE). After that, ACMP was electropolymerized onto the surface of GN-modified GCE (GN/GCE), constructing a poly- ACMP and GN composite film-modified GCE (pACMP/GN/GCE). The oxidation behavior of SY was examined, and an independent oxidation wave was observed. Compared with the bare GCE, the GN/GCE and the poly-ACMP modified GCE (pACMP/GCE) apparently increased the oxidation peak current of SY. Interestingly, the polypyrimidine and GN 
exhibited synergetic enhancement effects toward the oxidation of SY, and further enhanced their oxidation signals greatly. Hence, a new highly sensitive and rapid electrochemical method was established for SY determination and softdrink sample analysis.

\section{Experimental}

Reagents and Chemicals. All chemicals were of analytical grade and used directly. ACMP was synthesized according to the reported procedure with some modifications [10]. Graphite powder (spectral pure) and DMF were purchased from Sinopharm Chemical Regent Co., Ltd (Shanghai, China). SY was obtained from Aladdin Industrial Corporation (Shanghai, China). Phosphate buffer solutions (PBS) were prepared from $\mathrm{KH}_{2} \mathrm{PO}_{4}$ and $\mathrm{Na}_{2} \mathrm{HPO}_{4}$. Ultrapure water was obtained from a Milli-Q water purification system.

Instruments. Electrochemical measurements were performed on a $\mathrm{CHI} 660 \mathrm{E}$ electrochemical workstation (Chenhua Instrument, Shanghai, China). Field emission scanning electron microscope (FE-SEM) images were recorded with a ZEISS Sigma FE-SEM (Carl Zeiss, Oberkochen, Germany). A GCE, a saturated calomel electrode, and a Pt wire were used as the working, reference, and counter electrodes, respectively.

Preparation of GN Suspension. The graphene suspension was prepared by sonicating the mixtures of graphite powder and sodium citrate in DMF solvent [11]. Typically, $15 \mathrm{mg}$ of graphite powder and $5 \mathrm{mg}$ of sodium gallate were added into $5 \mathrm{~mL}$ of DMF, and then sonicated in a KQ-2200DE ultrasonicator (frequency: $50 \mathrm{kHz}$, power: $220 \mathrm{~W}$; Kunshan Ultrasonic Instrument Co., $\mathrm{Ltd}$, China) for $3 \mathrm{~h}$ at room temperature. Thus, $3.0 \mathrm{mg} \cdot \mathrm{mL}^{-1} \mathrm{GN}$ suspension was obtained.

Electrode Modification. A GCE with diameter of $3 \mathrm{~mm}$ was polished with $0.05 \mathrm{~mm}$ alumina slurry and then rinsed and sonicated with $\mathrm{HNO}_{3}(1: 1)$, ethanol and water for $5 \mathrm{~min}$. Firstly, $5 \mu \mathrm{L}$ of the resulting graphene suspension was coated on the GCE surface, and then dried at $45^{\circ} \mathrm{C}$ in an oven. After that, electropolymerization of ACMP on the GN/GCE was carried out by successive potential sweeps between $0.20 \mathrm{~V}$ and $1.60 \mathrm{~V}$ at a scan rate of $50 \mathrm{mV} \cdot \mathrm{s}^{-1}$ in $0.10 \mathrm{~mol} \cdot \mathrm{L}^{-1} \mathrm{NaOH}$ containing $1.0 \times 10^{-3} \mathrm{~mol} \cdot \mathrm{L}^{-1}$ ACMP. Then, the modified electrode surface was thoroughly washed with ultrapure water to remove any adsorbed species. Finally, the resulting nanocomposite showed strong signal enhancement effects for SY detection.

Analytical Procedure. Unless otherwise noted, $0.1 \mathrm{~mol} \cdot \mathrm{L}^{-1} \mathrm{PBS}$ at $\mathrm{pH} 6.5$ was used as supporting electrolyte for SY detection. After 80-s accumulation at $0 \mathrm{~V}$, the square wave voltammetries (SWVs) were recorded from 0.4 to $0.9 \mathrm{~V}$, and the oxidation peak current at $0.71 \mathrm{~V}$ was used for SY determination. The amplitude was $25 \mathrm{mV}$, and frequency was $15 \mathrm{~Hz}$.

\section{Results and Discussion}

The surface morphologies of different modified GCEs were characterized by SEM. The surface of GN/GCE was coated by flaky sheets, indicating that graphite was exfoliated into graphene nanosheets (Fig. 1A). After ACMP was electropolymerized on the surface of GN/GCE, numerous sticks with sizes of several micrometers was observed on the surface, as depicted in Fig. 1B. These sticks further enhanced the surface roughness of GN/GCE, and provided more active sites. Therefore, the pACMP and GN composite film exhibited much stronger accumulation abilities, resulting in higher electrochemical activity. 

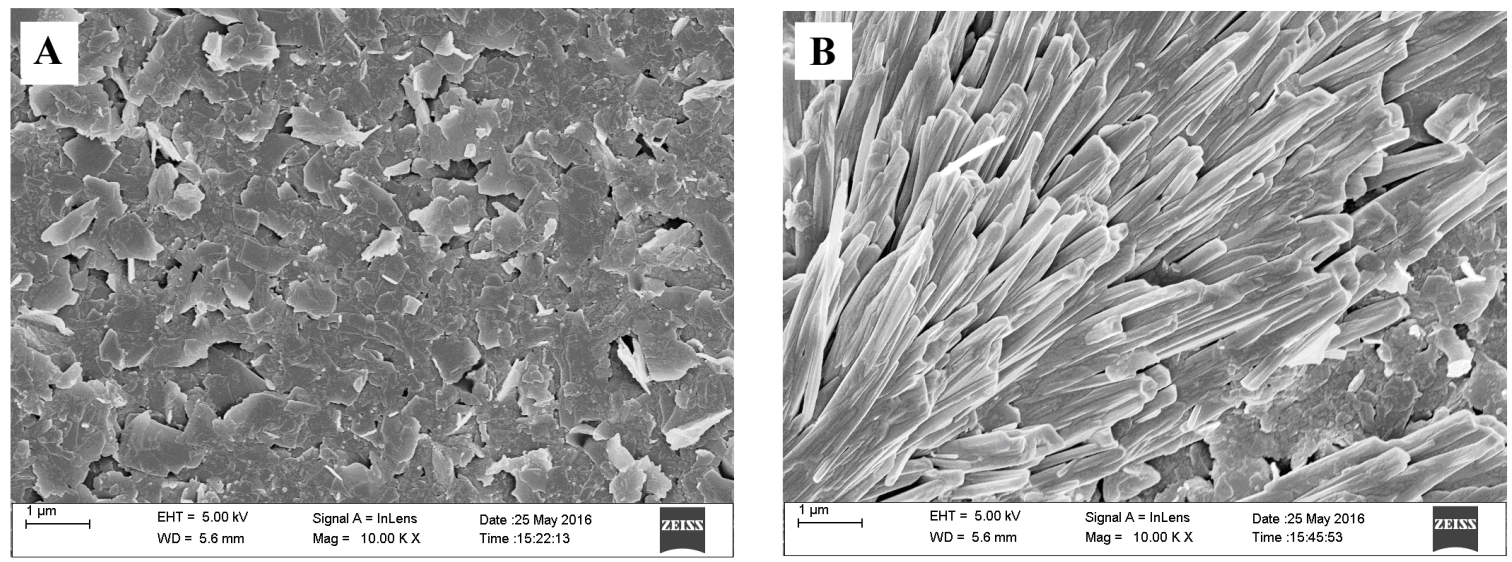

Fig. 1. FE-SEM images of GN/GCE (A) and pACMP/GN/GCE (B).

The electrochemical behaviors of SY on different GCEs were investigated using cyclic voltammetry $(\mathrm{CV})$, and the results are shown in Fig. 2. One oxidation wave with extremely low peak currents was observed on the surface of bare GCE (curve a), suggesting that the electrochemical activity of SY was very low on the bare GCE surface. However, a pair of redox peaks appeared on the surfaces of pACMP/GCE (curve b), GN/GCE (curve c) and pACMP/GN/GCE (curve d). The oxidation peak potential for SY was $0.714 \mathrm{~V}$, and the reduction peak potential was $0.729 \mathrm{~V}$. The appearance of sensitive redox waves and the greatly-enhanced peak currents manifested that the as-prepared modified electrodes, especially pACMP/GN/GCE, obviously improved the electrochemical activity of SY.

The SWVs of the composite film-modified electrode (pACMP/GN/GCE) under the optimized conditions at different concentrations of SY $\left(0.003-5.4 \mu \mathrm{mol} \cdot \mathrm{L}^{-1}\right)$ were recorded (Fig. 3A). As illustrated in Fig. 3B, the regression equation was $I_{\mathrm{p}}=-0.1896+3.6825 c\left(I_{\mathrm{p}}\right.$ in $\mu \mathrm{A}, c$ in $\mu \mathrm{mol} \cdot \mathrm{L}^{-1}$, and $\mathrm{R}=0.9994)$ for $\mathrm{SY}(\mathrm{S} / \mathrm{N}=3)$. Electrochemical performance of the as-prepared electrode to detect $\mathrm{SY}$ was compared with previous works, the merits of analytical characteristics were summarized in Table 1.

SY in softdrink samples was tested with the proposed film-modified electrode using the calibration method to demonstrate the applicability of the proposed method. The results are described in Table 2. The recoveries were in the range of $95.8 \%-104.6 \%$, which indicated the applicability and reliability of this method. 


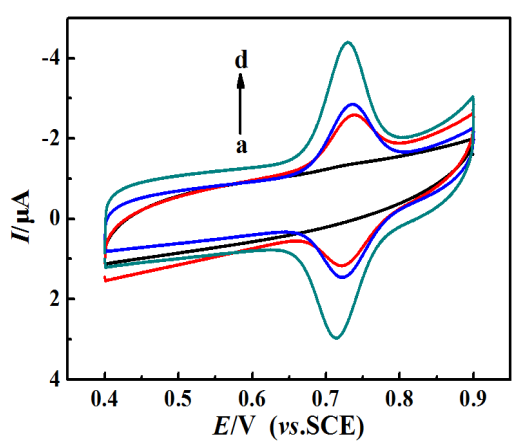

Fig. 2. $\mathrm{CV}$ curves of $3 \mu \mathrm{mol} \cdot \mathrm{L}^{-1}$ $\mathrm{SY}$ in PBS $(\mathrm{pH}=6.5)$ on GCE (a), pACMP/GCE (b), GN/GCE (c), and pACMP/GN/GCE (d).
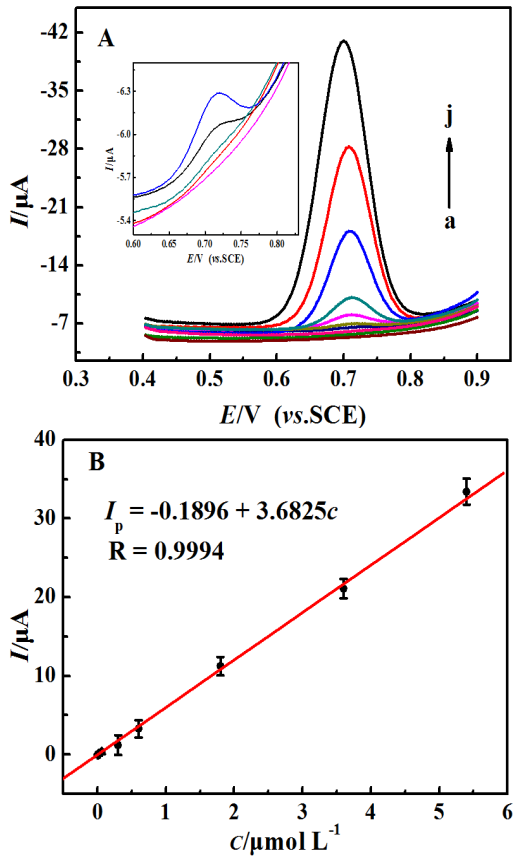

Fig. 3. (A) SWV curves of $\mathrm{pACMP} / \mathrm{GN} / \mathrm{GCE}$ in $\mathrm{PBS}(\mathrm{pH}$ $6.5)$ at different concentrations of $\mathrm{SY}(\mathrm{a} \rightarrow \mathrm{j}: 0,0.003,0.006$, $0.03,0.06,0.3,0.6,1.8,3.6$, and $\left.5.4 \mu \mathrm{mol} \cdot \mathrm{L}^{-1}\right) ;(\mathrm{B})$ Calibration plots of SY.

Table 1. Comparison of different electrodes for SY determination.

\begin{tabular}{lllll}
\hline Entry & Electrode materials & $\begin{array}{l}\text { Linear range } \\
{\left[\mu \mathrm{mol} \cdot \mathrm{L}^{-1}\right]}\end{array}$ & $\begin{array}{l}\text { Detection limit } \\
{\left[\mu \mathrm{mol} \cdot \mathrm{L}^{-1}\right]}\end{array}$ & References \\
\hline 1 & $\mathrm{TiO}_{2}$-graphene/CPE & $0.02-2.05$ & 0.006 & 7 \\
2 & $\mathrm{GN}-\mathrm{PTA} / \mathrm{GCE}$ & $0.0332-0.464$ & 0.0011 & 8 \\
3 & Ni-graphene/GCE & $0.0074-0.44$ & 0.002 & 12 \\
4 & Expanded Graphite/CPE & $0.05-2$ & 0.005 & 13 \\
5 & MWCNT/GCE & $0.055-11$ & 0.022 & 14 \\
6 & Graphene/GCE & $0.0025-0.25$ & 0.001 & 15 \\
7 & MWCNTs-IL/CCE & $0.4-110$ & 0.1 & 16 \\
8 & $\mathrm{pACMP/GN/GCE}$ & $0.003-5.4$ & 0.00095 & This work \\
\hline
\end{tabular}

Table 2. The determination of SY in softdrink samples.

\begin{tabular}{lllll}
\hline Sample & Added $\left[\mu \mathrm{mol} \cdot \mathrm{L}^{-1}\right]$ & Found $\left[\mu \mathrm{mol} \cdot \mathrm{L}^{-1}\right]$ & Recovery $[\%]$ & RSD [\%] \\
\hline 1 & 0.5 & 0.479 & 95.8 & 4.2 \\
2 & 1.0 & 1.046 & 104.6 & 4.6 \\
3 & 2.0 & 2.050 & 102.5 & 2.5 \\
4 & 3.0 & 2.890 & 96.3 & 3.7 \\
\hline
\end{tabular}

\section{Conclusions}

In this work, graphene was facilely obtained via one-step solvent exfoliation of graphite powder based on organic salt assistance in common organic solvent. A graphene/polypyrimidine composite film-modified electrode was conveniently fabricated by drop and electropolymerization method. The resulting electrode demonstrated synergetic signal enhancement toward the oxidation of SY. On the basis of this, a new electrochemical sensor was successfully constructed for SY detection, which possessed apparently higher sensitivity and lower detection limit (Table 1). This presented method 
displayed high sensitivity, rapid response, and good handling convenience with numerous potential application.

\section{Acknowledgements}

This work was financially supported by the National Natural Science Foundation of China (21561011), and Scientific \& Technological Innovation Team Project of Hubei University for Nationalities (MY2014T004).

\section{References}

[1] S. Haldar, R.G. Amorim, B. Sanyal, R.H. Scheichera and A.R. Rochac: RSC Adv. Vol. 6 (2016), p. 6702

[2] S. Deng and V. Berry: Mater. Today Vol. 19 (2016), p. 197

[3] K.J. Huang, H.L. Shuai and J.Z. Zhang: Biosens. Bioelectron. Vol. 77 (2016), p. 69

[4] Y.S. Gao, J.K. Xu, L.M. Lu, L.P. Wu, K.X. Zhang, T. Nie, X.F. Zhu and Y. Wu: Biosens. Bioelectron. Vol. 62 (2014), p. 261

[5] J. Baek, J. Lee, M. Joo, D. Han, H. Kim, H. Seong, J. Lee, J. Kim, S. Yoo, S. Jeon and S. Gaplm: J. Mater. Chem. C Vol. 4 (2016), p. 831

[6] H. Bai, S. Wang, P. Liu, C. Xiong, K. Zhang and Q. Cao: J. Electroanal. Chem. Vol. 771 (2016), p. 29

[7] T. Gan, J. Sun, W. Meng, L. Song and Y. Zhang: Food Chem. Vol. 141 (2013), p. 3731

[8] T. Gan, J. Sun, S. Cao, F. Gao, Y. Zhang and Y. Yang: Electrochim. Acta Vol. 74 (2012), p. 151

[9] X. Ye, Y. Du, D. Lu and C. Wang: Anal. Chim. Acta Vol. 779 (2013), p. 22

[10] A.A. Fadda, E.A. El-Latif, S. Bondock and A. Samir: Synth. Commun. Vol. 38 (2008), p. 4352

[11]J. Fu, X.H. Tan, Z. Shi, X.J. Song and S.H. Zhang: Electroanal. Vol. 28 (2016), p. 203

[12]T. Gan, J.Y. Sun, Q. Wu, Q.S. Jing and S. Yu: Electroanal. Vol. 25 (2013), p. 1505

[13] J. Zhang, H.H. Zhu, M.L. Wang, W.C. Wang and Z.D. Chen: J. Electrochem. Soc. Vol. 160 (2013), p. H459

[14] W.K. Zhang, T. Liu, X.J. Zheng, W.S. Huang and C.D. Wan: Colloid. Surface B Vol. 74 (2009), p. 28

[15]C. Wu, Q. Cheng, K.B. Wu, G. Wu and Q. Li: Anal. Chim. Acta Vol. 825 (2014), p. 26

[16] M.R. Majidi, R.F.B. Baj and A. Naseri: Food Anal. Methods Vol. 6 (2013), p. 1388 